EPJ Web of Conferences 61, 07010 (2013)

DOI: $10.1051 /$ epjconf/ 20136107010

(C) Owned by the authors, published by EDP Sciences, 2013

\title{
A large rotation of the polarization angle of the TeV Blazar W Comae
}

\author{
Erika Benítez $^{1, a}$, Marco Sorcia ${ }^{1, b}$, and David Hiriart ${ }^{2, c}$ \\ ${ }^{1}$ Instituto de Astronomía, Universidad Nacional Autónoma de México, AP 70-264, 04510 DF, Mexico \\ ${ }^{2}$ Instituto de Astronomía, Universidad Nacional Autónoma de México, AP 877, 22800, Ensenada BC, Mexico
}

\begin{abstract}
Optical R-band polarimetric variability observations of the TeV Blazar W Comae are presented. Data obtained from 2008 February to 2013 May ( 5.2 years) are analyzed. The source presented a maximum flux variability of $\sim 3 \mathrm{mJy}$. The minimum variability time scale displayed by the source during the monitored period is $\Delta t \sim 3.3$ d. A maximum linear polarisation degree value of $\mathrm{P}=(33.8 \pm 1.6) \%$ was observed in 2013 May 12 . A rotation of the position angle from $78^{\circ}$ (2008 March 10) to $229^{\circ}$ (2008 July 11) was observed. This rotation corresponds to a large change of $\Delta \theta \sim 150^{\circ}$ in a period of $123 \mathrm{~d}$ or to a rotation of $\sim 1.2^{\circ}$ per day. After the high activity state observed in 2008 , the position angle shows a preferential value of $\sim 56^{\circ}$, with variations of $\sim 60-120^{\circ}$. From the Stokes parameters we infer the existence of two optically-thin synchrotron components that contribute to the optical polarized flux. One of them is stable, with $\mathrm{P} \sim 11 \%$.
\end{abstract}

\section{Introduction}

The TeV-blazar W Comae at $\mathrm{z}=0.102$ (also known as $\mathrm{ON}$ 231 or B1219+285) was discovered as a radio source by [1]. For more than forty years this source has been monitored in the optical bands. Its optical historical lightcurve has been followed since 1897 when it was known as a variable star [2]. A historical maximum of $R=12.12$ mag was observed during an outburst in 1998 April 23 [see 3]. Multiband optical polarization observations were also done by these authors, just before and during the brightest phase (1998 April 17-25). They found that during the brightest state, the polarization was low in the UBV filters ( $\sim 2$ to $4 \%$ ) and less than $0.4 \%$ in the $R_{c}$ and $I_{c}$ filters.

On the other hand, the $\gamma$-ray emission of W Comae was first detected by the Energetic Gamma Ray Experiment Telescope (EGRET) on board the Compton Gamma Ray Observatory (CGRO) in the $100 \mathrm{MeV}$ to $10 \mathrm{GeV}$ band [4]. Later, BeppoSAX data analysis of W Comae given by [5] show that this source can be considered as an intermediate peaked BL Lac object (IBL) i.e., between the high peaked BL Lac (HBL) and low-peaked BL Lac (LBL) sources. W Comae was discovered to be a very high energy (VHE) $\gamma$-ray emitter by VERITAS in 2008 March 15 [see 6], therefore it is the first IBL detected at VHE. A subsequent multiwavelength campaign on this object was coordinated during a major $\gamma$-ray flare in 2008 June [7].

In this work some relevant results on the optical photopolarimetric monitoring done to the TeV blazar W Comae are presented. Data collected during the monitoring period allowed us to establish some long and short-term

\footnotetext{
a e-mail: erika@astro.unam.mx

be-mail: msorcia@astro.unam.mx

ce-mail: hiriart@astrosen.unam.mx
}

variability properties of the polarized emission of this object in the $R$-band.

\section{Observations}

R-band photopolarimetric observations of W Comae were done using the $84 \mathrm{~cm}$ telescope at the San Pedro Mártir Observatory (SPM) in Baja California Mexico, between 2008 February 28 (JD 2,454,524) and 2013 May 17 (JD $2,456,429)$. The instrument POLIMA was attached to the telescope which is a direct image polarimeter. During this period, 32 runs of seven nights per run around the new moon phase were done, in total we collected 141 data points. Photometric data on this source obtained in 2008 are already published in [7]. In this work all photometric and polarimetric data collected up to now are presented. The differential $R$-band magnitudes of W Comae were calculated using the standard star A distant about $\sim 1.2$ arc-minutes to the South-East from the study object. The magnitude of the comparison star $\mathrm{A}$ in the $R-$ band is (11.72 \pm 0.04$)$ mag [8]. The exposure time was $80 \mathrm{~s}$ per image for W Comae. Polarimetric calibrations were done using the polarized standard stars ViCyg12 and HD155197 and the unpolarized standard stars GD319 and $\mathrm{BD}+332642$ [9]. The $R$-band magnitudes were corrected for the host galaxy contribution, $m_{R(\text { host })}=16.60$, fitting a de Vaucouleaurs profile [see 10]. Then, the magnitudes were converted into apparent fluxes using the expression: $F_{\text {obs }}=K_{0} \times 10^{-0.4 m_{R}}$, with $K_{0}=3.08 \times 10^{6} \mathrm{mJy}$ [11], for an effective wavelength of $\lambda=640 \mathrm{~nm}$. Also, all data were corrected for the $180^{\circ}$ ambiguity in the polarization angle in a way that the differences observed between the polarization angle of temporal adjacent data should be less that 
$90^{\circ}$. More details on the reduction process, error estimations and statistical analysis can be found in [12].

Figure 1 shows the R-band light curve (LC), the polarization degree and the PA obtained from the analysis of all data points. For clarity in the discussion, the entire period of observations has been divided into six main cycles: Cycle I from 2008 February 28 to 2008 July 11 JD (2,454,524 to 2,454,658); Cycle II from 2009 March 24 to 2009 May 28 JD $(2,454,914$ to $2,454,979)$; Cycle III from 2009 November 14 to 2010 June 16 JD $(2,455,150$ to 2,455,363); Cycle IV from 2011 January 11 to 2011 June 4 JD (2,455,572 to 2,455,716); Cycle V from 2011 December 15 to 2012 June 1 JD $(2,455,911$ to $2,456,079)$, and Cycle VI from 2013 January 13 to 2013 May 17 JD $(2,456,306$ to $2,456,429)$. These cycles are marked with dashed vertical lines.

\section{Results}

Figure 2 shows the correlations between the flux and the polarization degree (top panel) and the flux and the polarization angle (bottom panel) for all cycles (see [12] for more details). To establish a possible correlation between the polarization degree and the $R$-band flux, a Pearson correlation coefficient was calculated $\left(\mathrm{r}_{F-p}\right)$. Using all data, we found that there is no correlation between the $R$-band flux and the polarization degree (see top panel of Figure 2). However, there is a slight tendency to increase the degree of polarization as the brightness decreases. We did not find any correlation between the $R$-band brightness and polarization degree in all cycles, except for the cycles II, III, and VI where a moderate anticorrelation exists. In Cycle II, the Pearson correlation coefficient is $\mathbf{r}_{F-p}$ $=-0.88 \pm 0.24$ during the fall of the flare. In Cycle III its value is $\mathrm{r}_{F-p}=-0.82 \pm 0.09$ during the rise of the flare. In cycle VI, $\mathrm{r}_{F-p}=-0.89 \pm 0.04$ (taking into account the rise and fall of the flare). This result points out that the flux and the polarization degree show a tendency to be anti correlated in periods of time $\sim$ weeks to months. On the other hand, a positive correlation of $r_{F-p}=0.93 \pm 0.11$ is found during Cycle I (2008 June 3-7 flare). In general, the polarization degree showed a random variability behavior, with a maximum and a minimum of $(33.8 \pm 1.6) \%(2013$ May 12, JD 2,456,424) and $(2.4 \pm 1.3) \%$ (2011 March 2, JD 2,455,622), respectively. The maximum variability observed was $\Delta P=31.4 \%$, in $\Delta t=802 \mathrm{~d}$ (or $2.2 \mathrm{yr}$ ). It is important to notice that the maximum value of the polarization degree occurred in Cycle VI, when the brightness was in its minimum value. In Cycle I, the maximum variability observed of the polarization degree is $\Delta P=11.1 \%$ in $\Delta t$ $=95 \mathrm{~d}$; in Cycle II, $\Delta P=14.7 \%$ in $\Delta t=6 \mathrm{~d}$; in Cycle III, $\Delta P=7.8 \%$ in $\Delta t=57 \mathrm{~d}$; in Cycle IV, $\Delta P=14.5 \%$ in $\Delta t=92$ $\mathrm{d}$; in Cycle V, $\Delta P=17.8 \%$ in $\Delta t=39 \mathrm{~d}$; and in Cycle VI, $\Delta P=28.5 \%$ in $\Delta t=55 \mathrm{~d}$.

In general our data do not show a clear correlation between the polarization angle and the $R$-band flux. Rather, the polarization angle tends to a preferential position of $\sim 56^{\circ}$ with respect to the flux variations, with variations of $\sim 15^{\circ}-59^{\circ}$ (see bottom panel of Figure 1). Nevertheless, when the source is brightening the PA varies in the range of $\sim 60^{\circ}-120^{\circ}$. In Cycle I a gradual rotation of the PA of $78^{\circ}$ (JD 2,454,535) to $229^{\circ}$ (JD 2,454,658) is observed. This correspond to a total rotation of $\sim 150^{\circ}$ in a period of 123 days (giving an average rate of rotation of $\sim 1.2^{\circ}$ per day). During the 2008 June 4 flare, the $P A=121^{\circ}$ continues its rising until a maximum of $229^{\circ}$ at the end of this cycle is reached. From our observations we have found that $\mathrm{W}$ Comae shows, in general, a random behavior. This has been explained by [see, e.g. 13] as due to the presence of one or more variable polarization components superimposed to a stable one. To identify the presence of a stable polarized component, we used the method explained in [12]. For the case of a two-component model, we define the average values of $Q$ and $U$ as the stable polarization component. From our data, the derived average values of the absolute Stokes parameters are $\langle Q\rangle=-0.22 \pm 0.02 \mathrm{mJy}$ and $\langle U\rangle=0.21 \pm 0.03 \mathrm{mJy}$ (see Figure 3 ). These average values correspond to a stable component with constant polarization degree $P_{c}=10.7 \pm 0.8 \%$ and polarization angle $\Theta_{c}=65^{\circ} \pm 2^{\circ}$. The constant polarization degree has a dispersion of $\sigma_{P_{c}}=6.4 \%$.

To estimate the polarization variable component parameters, we looked for a possible linear relation between $Q$ versus $I$ and $U$ versus $I$ for the six relevant cycles [see, 14]. For Cycle IV no linear correlation between these parameters was found; rather, they appear to be randomly related. In contrast, for Cycles I, II, III, V, and VI, our data show a linear tendency between these parameters. We made a least square fit to the data in order to find the slopes and the linear correlation coefficients $r_{Q I}$ and $r_{U I}$. Figure 4 shows this linear correlations between the Stokes parameters for Cycle I. The maximum polarization degree found for the variable component is $p_{\text {var }}=(40.1 \pm 5.1) \%$, with a polarization angle $\theta_{\text {var }}=-64^{\circ} \pm 7^{\circ}$. From the above results, we infer the presence of a stable component that we assume associated with the relativistic jet, and also a variable component that can be related to the propagation of the shock. Therefore, the observed polarization would be the result of the superposition of these two optically thin synchrotron components. From equations (16) and (17) in [12], and using the previously found parameters $P_{\mathrm{c}}$ and $\Theta_{\mathrm{c}}$ as the parameters for the constant component $p_{\text {cons }}$ and $\theta_{\text {cons }}$, respectively, we found the values of the polarized variable component $p_{v a r}, \theta_{v a r}$ and $I_{v a r}$ for each night, where $I_{v a r}$ is the intensity flux of the polarized variable component. In Figure 5 the observed polarimetric parameters (empty squares) are shown as a combination of the variable (solid dots) and constant (dashed line) component for Cycle V. Also it can be noted that the variable polarized component dominates over the constant one, and this happens during the whole monitored period.

\section{Summary}

From our data, a minimum time-scale variations for W Comae of $\sim 3 \mathrm{~d}$ is found. These variations are superimposed to a longer-term flare of $\sim 3$ months that have a period of $\sim 0.9 \mathrm{y}$, in agreement to [15] and [3]. 


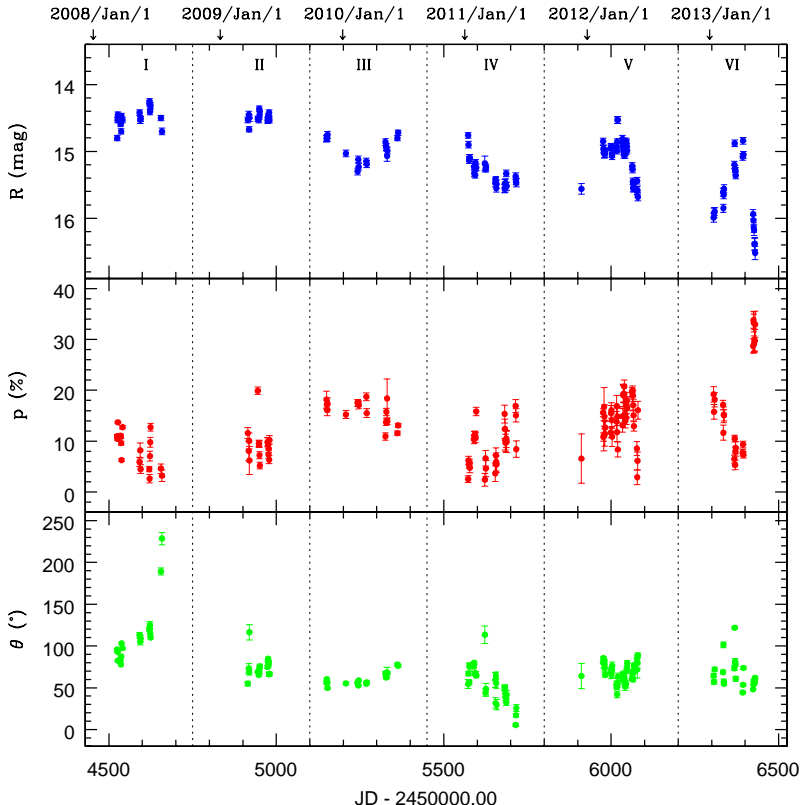

Figure 1. Light curve of W Comae obtained in $\sim 5.2$ years. Upper panel shows the R-band flux variations, middle panel the polarization degree $\mathrm{P} \%$ variations, and lower panel the position angle variations $(\theta)$. During the outburst of 2008 , represented by Cycle I (2008 Feb 29 to Jul 11) the position angle $\theta$ varied towards larger values. Then, in Cycle II (2009 March 24 to May 28 ) the value of $\theta$ declines more rapidly by a factor of $\sim 4$. From Cycles III to VI the source presented a preferential position angle of optical polarization PA $\sim 56^{\circ}$. In Cycle VI (2013 Jan 13 to May 17) we find the minimum flux value ( $0.76 \mathrm{mJy})$ and also the maximum value $\mathrm{P} \sim 34 \%$.

The polarimetric analysis led us to infer the superposition of two optically-thin synchrotron components. In 2008 June 8 a large flare $(>200 \mathrm{GeV}$ ) was detected with VERITAS in W Comae with a significance of 10.3 [7]. Data from our monitoring from 2008 June 4-7 show an increase in the $R$-band flux. Unfortunately, due to bad weather we could not obtained data from June 8, when the maximum brightness was observed in the $\gamma$-rays. However, our data show a gradual increase in the value of the PA from $78^{\circ}$ a $229^{\circ} \mathrm{JD}(2,454,535-2454,658)$ and a large rotation of $\sim 150^{\circ}$ during Cycle I, coinciding with the 2008 flare. The large rotation of the PA observed during the 2008 outburst can be interpreted as due to an asymmetric distribution of the magnetic filed with respect to the jet axis. According to [16], the VLBI structure of W Comae shows a core with a multicomponent jet elongated with a position angle $\mathrm{PA} \sim 110^{\circ}$ and a spiral structure. Therefore, we suggest that the rotation can be produced by a swing of the jet along the visual line of sight, or a curved trajectory of the dissipation/emission pattern.

\section{Acknowledgements}

E.B., M. S. and D.H. thank financial support from UNAMDGAPA-PAPIIT through grant IN116211-3.

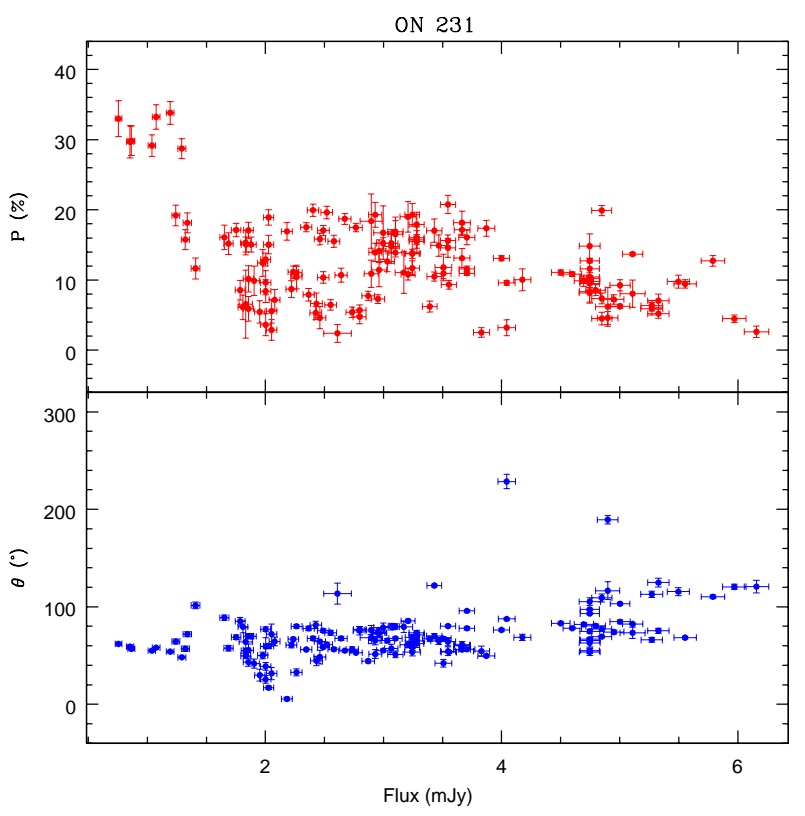

Figure 2. Upper panel shows variations between the polarization degree (P\%) and flux; lower panel the variations between PA $(\theta)$ vs flux. No correlation is found between the $R$-band flux and the polarization degree.

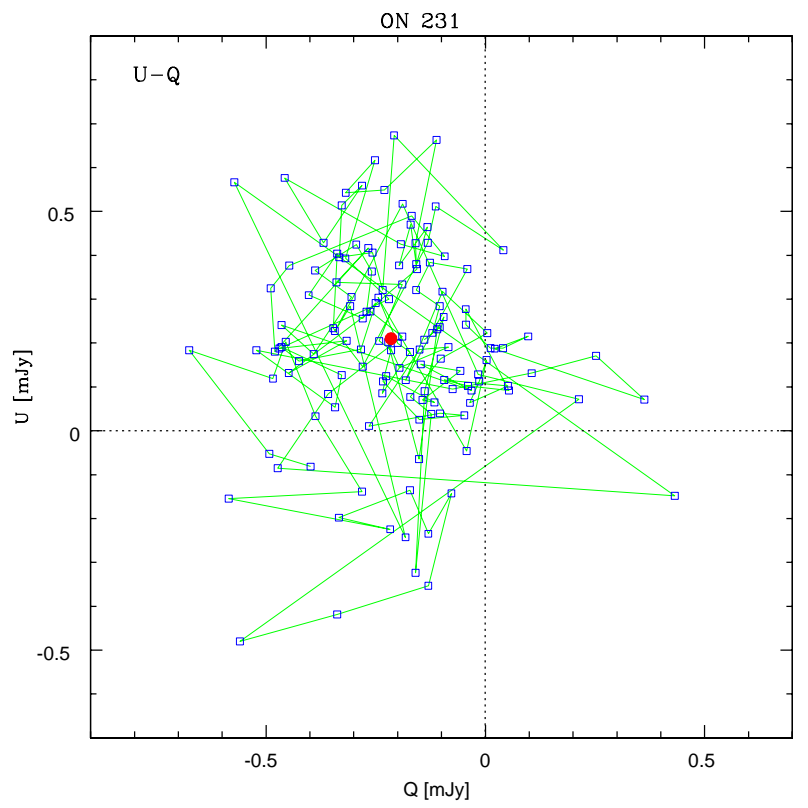

Figure 3. Q vs U plane for the stable polarized component. The obtained values for the absolute Stokes parameters are $\langle Q\rangle=$ $0.22 \pm 0.02 \mathrm{mJy}$ and $\langle U\rangle=0.21 \pm 0.03 \mathrm{mJy}$. This average value is represented by the red-filled point in the plane. 


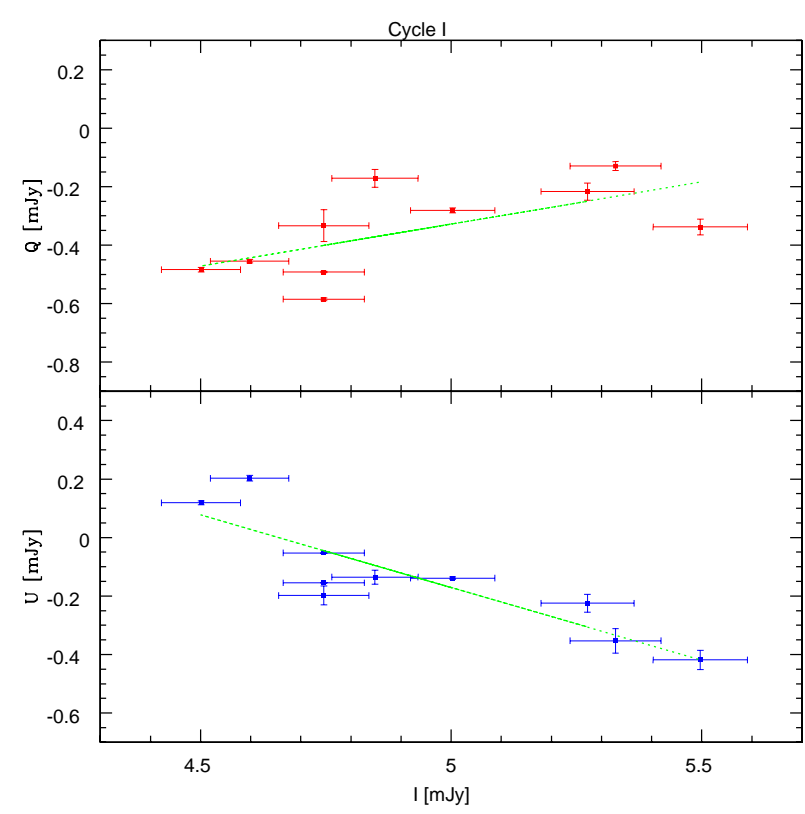

Figure 4. Correlations between the Stokes parameters Q and $\mathrm{U}$ vs I during Cycle I. From these correlations we estimated the polarimetric parameters of the variable polarized component, and find that its maximum value is $P_{v a r}=(40.1 \pm 5.1) \%$

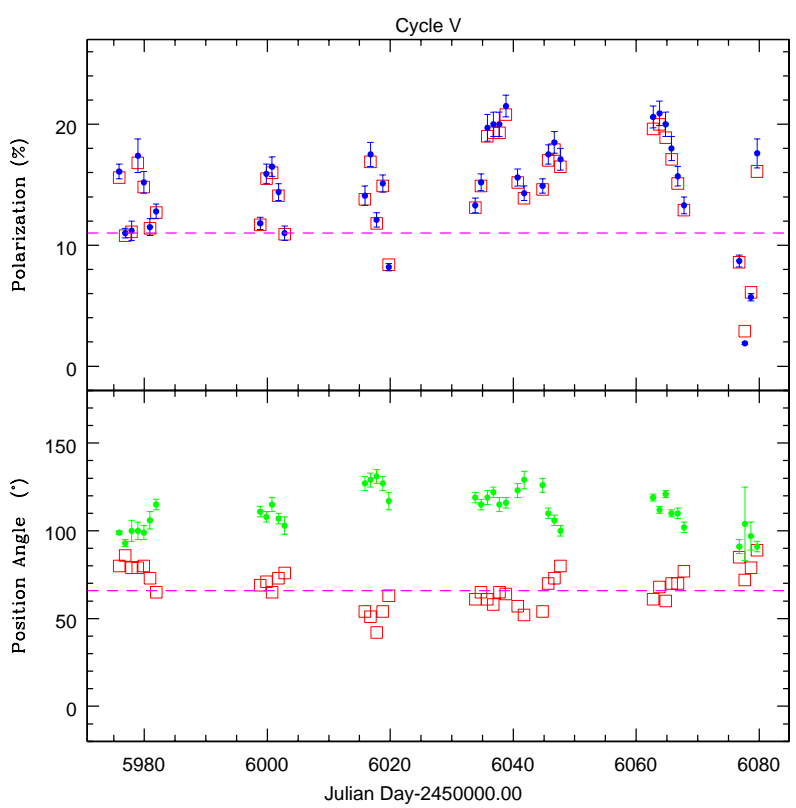

Figure 5. Variations of the two polarized components during Cycle V. Red empty squares represent the observations. Dashed lines mark the values of the polarized degree (upper panel) and the position angle $\theta$ (lower panel) of the constant polarized component. Blue and green-filled dots represent the variations of the variable polarized component.

\section{References}

[1] I.W.A. Browne, Nature 231, 515 (1971)

[2] M. Wolf, Astronomische Nachrichten 202, 415 (1916)

[3] E. Massaro, M. Maesano, F. Montagni, R. Nesci, G. Tosti, M. Fiorucci, M. Luciani, L.O. Takalo, A. Sillanpää, S. Katajainen et al., Astronomy and Astrophysics 342, L49 (1999)

[4] R.C. Hartman, D.L. Bertsch, S.D. Bloom, A.W. Chen, P. Deines-Jones, J.A. Esposito, C.E. Fichtel, D.P. Friedlander, S.D. Hunter, L.M. McDonald et al., Astrophysical Journal Supplement 123, 79 (1999)

[5] G. Tagliaferri, G. Ghisellini, P. Giommi, L. Chiappetti, L. Maraschi, A. Celotti, M. Chiaberge, G. Fossati, E. Massaro, M. Maesano et al., Astronomy and Astrophysics 354, 431 (2000)

[6] V.A. Acciari, E. Aliu, M. Beilicke, W. Benbow, M. Böttcher, S.M. Bradbury, J.H. Buckley, V. Bugaev, Y. Butt, O. Celik et al., Astrophysical Journal Letter 684, L73 (2008)

[7] V.A. Acciari, E. Aliu, T. Aune, M. Beilicke, W. Benbow, M. Böttcher, D. Boltuch, J.H. Buckley, S.M. Bradbury, V. Bugaev et al., Astrophysical Journal 707, 612 (2009)

[8] M. Fiorucci, G. Tosti, Astronomy and Astrophysics Supplement 116, 403 (1996)

[9] G.D. Schmidt, R. Elston, O.L. Lupie, Astronomical Journal 104, 1563 (1992)

[10] K. Nilsson, T. Pursimo, J. Heidt, L.O. Takalo, A. Sillanpää, W. Brinkmann, Astronomy and Astrophysics 400, 95 (2003)

[11] K. Nilsson, M. Pasanen, L.O. Takalo, E. Lindfors, A. Berdyugin, S. Ciprini, J. Pforr, Astronomy and Astrophysics 475, 199 (2007)

[12] M. Sorcia, E. Benítez, D. Hiriart, J.M. López, J.I. Cabrera, R. Mújica, J. Heidt, I. Agudo, K. Nilsson, M. Mommert, Astrophysical Journal Supplement 206, 11 (2013)

[13] T.W. Jones, L. Rudnick, H.D. Aller, M.F. Aller, P.E. Hodge, R.L. Fiedler, Astrophysical Journal 290, 627 (1985)

[14] V.A. Hagen-Thorn, V.M. Larionov, S.G. Jorstad, A.A. Arkharov, E.I. Hagen-Thorn, N.V. Efimova, L.V. Larionova, A.P. Marscher, Astrophysical Journal 672, 40 (2008)

[15] G. Tosti, M. Fiorucci, M. Luciani, N. Rizzi, M. Villata, C.M. Raiteri, G. de Francesco, L. Lanteri, M. Chiaberge, A. Peila et al., Astronomy and Astrophysics Supplement 130, 109 (1998)

[16] D. Weistrop, D.B. Shaffer, P. Hintzen, W. Romanishin, Astrophysical Journal 292, 614 (1985) 